\title{
Fourier-domain low-coherence interferometry for light-scattering spectroscopy
}

\author{
Adam Wax, Changhuei Yang, and Joseph A. Izatt \\ Department of Biomedical Engineering and the Fitzpatrick Center for Photonics, Duke University, Durham, North Carolina 27708
}

Received February 10, 2003

\begin{abstract}
We present a novel method for obtaining depth-resolved spectra for determining scatterer size through elasticscattering properties. Depth resolution is achieved with a white-light source in a Michelson interferometer with the mixed signal and reference fields dispersed by a spectrograph. The spectrum is Fourier transformed to yield the axial spatial cross correlation between the signal and reference fields with near 1- $\mu \mathrm{m}$ depth resolution. Spectral information is obtained by windowing to yield the scattering amplitude as a function of wave number. The technique is demonstrated by determination of the size of polystyrene microspheres in a subsurface layer with subwavelength accuracy. Application of the technique to probing the size of cell nuclei in living epithelial tissues is discussed. (C) 2003 Optical Society of America

OCIS codes: $170.4500,290.1350,120.3180$.
\end{abstract}

Light-scattering spectroscopy (LSS) has received much attention recently as a means of probing cellular morpholog $y^{1-3}$ and diagnosing dysplasia. ${ }^{4-6}$ LSS examines the elastic-scattering properties of cell organelles to infer their sizes. To measure cellular features in tissues, one must distinguish singly scattered light from diffuse light, which no longer carries easily accessible information about scatterers. This distinction is usually accomplished by polarization gating, ${ }^{1}$ by restricting studies to weakly scattering samples, ${ }^{2}$ or by using modeling to remove the diffuse component., ${ }^{4,5}$

Low-coherence interferometry (LCI) has been explored as an alternative for detecting singly scattered light from subsurface sites for LSS. Initial experiments with a broadband light source and its second harmonic showed that elastic-scattering information could be recovered with LCI. ${ }^{7}$ More recently, angle-resolved low-coherence interferometry was used to obtain structural information by examination of the angular distribution of scattered light. ${ }^{8,9}$ Angle-resolved LCI has been successfully applied to measuring cellular morphology $\mathrm{y}^{3}$ and to diagnosing intraepithelial neoplasia in an animal model of carcinogenesis. ${ }^{6}$ The latter study was significant because it demonstrated the strength of joining LSS and LCI as a biomedical diagnostic technique.

This Letter presents a new modality combining LSS and LCI as Fourier-domain low-coherence interferometry (fLCI). This new technique recovers depth-resolved structural information by Fourier transforming the spectrum of two mixed fields, as is done with spectral radar. ${ }^{10-12}$ In fLCI, this approach is used to obtain depth-resolved spectra of scattered light. The capabilities of fLCI are demonstrated below by extraction of the size of polystyrene beads in a subsurface layer. These results are discussed in the context of extending fLCI to probing the nuclear morphology of subsurface cell layers.

The fLCI scheme is shown in Fig. 1. White light from a tungsten source (6.5 W, Ocean Optics) is coupled into a multimode fiber $(200-\mu \mathrm{m}$ core diameter). The fiber output is collimated to produce a pencil beam (5-mm diameter). The white-light beam is split by beam splitter BS into a reference beam and an in- put beam to the sample. The light scattered by the sample is recombined at the beam splitter with light reflected by the reference mirror (M) and then coupled into a multimode fiber. The output of the fiber coincides with the input slit of a miniature spectrograph (USB2000, Ocean Optics), where the light is spectrally dispersed and detected.

The detected signal is linearly related to the intensity as a function of wavelength $I(\lambda)$, which can be related to the signal and reference fields $\left(E_{s}, E_{r}\right)$ as

$\langle I(\lambda)\rangle=\left\langle\left|E_{s}(\lambda)\right|^{2}\right\rangle+\left\langle\left|E_{r}(\lambda)\right|^{2}\right\rangle+2 \operatorname{Re}\left\langle E_{s}(\lambda) E_{r}^{*}(\lambda)\right\rangle \cos \phi$

where $\phi$ is the phase difference between the two fields and $\langle\ldots\rangle$ denotes an ensemble average. We extract the interference term by measuring the intensity of the signal and reference beams independently and subtracting them from the total intensity.

We obtain the axial spatial cross-correlation function, $\Gamma_{\mathrm{SR}}(z)$, between the sample and reference fields by rescaling the wavelength spectrum into a wave-number $(k=2 \pi / \lambda)$ spectrum and then Fourier transforming:

$$
\Gamma_{\mathrm{SR}}(z)=\int \mathrm{d} k \exp (i k z)\left\langle E_{s}(k) E_{r}{ }^{*}(k)\right\rangle \cos \phi .
$$

This term is an axial spatial cross correlation, related to the temporal or longitudinal coherence of the two

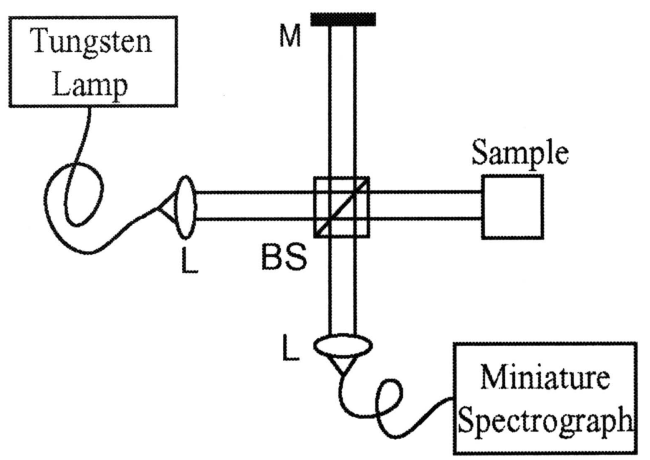

Fig. 1. Schematic of fLCI system. Ls, lenses. Other abbreviations defined in text. 
fields. Figure 2(a) shows the cross correlation between the signal and reference fields when the sample is a mirror, identical to the reference mirror. In this case, the fields are identical and the autocorrelation is given by the transform of the incident field spectrum, modeled as a Gaussian spectrum with center wave number $k_{0}=10.3 \mu \mathrm{m}^{-1}$ and $1 / e$ width $\Delta k_{1 / e}=2.04 \mu \mathrm{m}^{-1}$ [Fig. 2(b)]. From this autocorrelation, we determine the coherence length of the field, $l_{c}=1.21 \mu \mathrm{m}$. This is slightly larger than the calculated width of $l_{c}=2 / \Delta k_{1 / e}=0.98 \mu \mathrm{m}$; the discrepancy most likely is due to uncompensated dispersion effects. Note that rescaling the field into wave-number space is a nonlinear process that can skew the spectrum if the process is not properly executed. ${ }^{13}$ In data processing, we apply a cubic spline fit to the rescaled wave-number spectrum and then resample with even spacing. The resampled spectrum is then Fourier transformed to yield the spatial correlation of the sample. This processing is accomplished in real time with LabView software.

In the experiments the sample consists of a glass coverslip (thickness, $d \sim 200 \mu \mathrm{m}$ ) with polystyrene beads that have been dried from suspension onto the back surface $(1.55-\mu \mathrm{m}$ mean diameter, $3 \%$ variance). Thus we can write the field scattered by the sample as

$$
\begin{aligned}
E_{s}(k)= & E_{\text {front }}(k) \exp (i k \delta z)+E_{\text {back }}(k) \\
& \times \exp [i k(\delta z+n d)],
\end{aligned}
$$

where $E_{\text {front }}$ and $E_{\text {back }}$ denote the field scattered by the front and the back surfaces of the coverslip, respectively; $\delta z$ is the difference between the path length of the reference beam and that of the light reflected from the front surface, and $n$ is the index of refraction of the glass. The effect of the microspheres will appear in the $E_{\text {back }}$ term, as the beads are small and attached closely to the back surface. Upon substituting Eq. (3) into Eq. (2), we obtain a two-peak distribution with the width of the peaks given by the coherence length of the source.

To obtain spectroscopic information, we apply a Gaussian window to the interference term before Fourier transforming. This process is similar to spectroscopic optical coherence tomography, ${ }^{14,15}$ as we recover spectra for light scattered at a particular depth. The windowed interference term takes the form $\left\langle E_{s}(k) E_{r}{ }^{*}(k)\right\rangle \exp \left\{-\left[\left(k-k_{w}\right) / \Delta k_{w}\right]^{2}\right\}$. By selecting a window $\left(\Delta k_{w}\right.$ small $)$ that is narrow compared with the features of $E_{s}$ and $E_{k}$, we effectively obtain $\left\langle E_{s}\left(k_{w}\right) E_{r}{ }^{*}\left(k_{w}\right)\right\rangle$. In processing the data below, we use $\Delta k_{w}=0.12 \mu \mathrm{m}^{-1}$, which degrades the coherence length by a factor of 16.7 but affords us the opportunity to examine the strength of the scattering at 50 different wave numbers over the $6-\mu \mathrm{m}^{-1}$ span of usable spectrum.

Figure 3(a) and 3(b) show depth-resolved crosscorrelation reflection profiles of the coverslip sample before and after processing, respectively. Since these profiles are represented as cross-correlation functions, the plots are symmetric about $z=0$. Thus the front surface reflection for $z>0$ is paired with the backsurface reflection for $z<0$, and vice versa. The signal-to-noise ratio for the front-surface reflection is given by the Poisson noise of the array CCD in the spectrograph (signal-to-noise ratio, 250:1); however, the smaller reflection signal from the back surface suffers worse from noise (signal-to-noise ratio, 25:1). Note that the coverslip reflection introduces dispersion relative to the reference-arm reflection, generating multiple peaks in the reflection profile, as seen from Fig. 3(a). When the spectroscopic window is applied, only a single peak is seen for each surface; however, several dropouts appear as a result of aliasing of the signal.

To obtain the spectrum of the scattered light, we repeatedly apply the Gaussian window and increase the center wave number by $0.12 \mu \mathrm{m}^{-1}$ between the successive applications. In this way, we generate a spectroscopic depth-resolved reflection profile. Figures 4(a) and $4(\mathrm{~b})$ show the spectrum for light scattered from the front and back surfaces, respectively, when no microspheres are present. The reflection from the front surface appears as a slightly modulated version of the source spectrum. The spectrum of the reflection from the rear surface, however, has been significantly modified. Thus in Eq. (3) we now take $E_{\text {front }}(k)=E_{s}(k)$ and $E_{\text {back }}(k)=T(k) E_{s}(k)$, where $T(k)$ represents the transmission through the coverslip.

The light-scattering spectra when microspheres are on the back surface of the coverslip are shown in Figs. 4(a) (dashed curve) and 4(c). The reflected spectrum from the front surface has not changed significantly, as expected. However, the spectrum for the back surface is now modulated. We examine the scattering properties $S(k)$ of the microspheres by writing
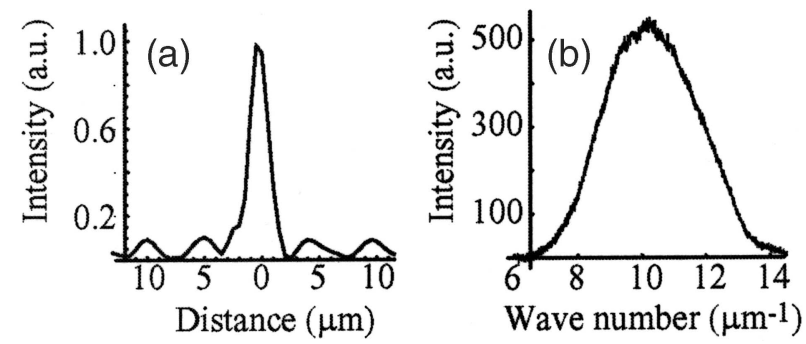

Fig. 2. Properties of white-light source. (a) Autocorrelation function showing coherence length $\left(l_{C}=1.2 \mu \mathrm{m}\right)$. (b) Spectrum of source.

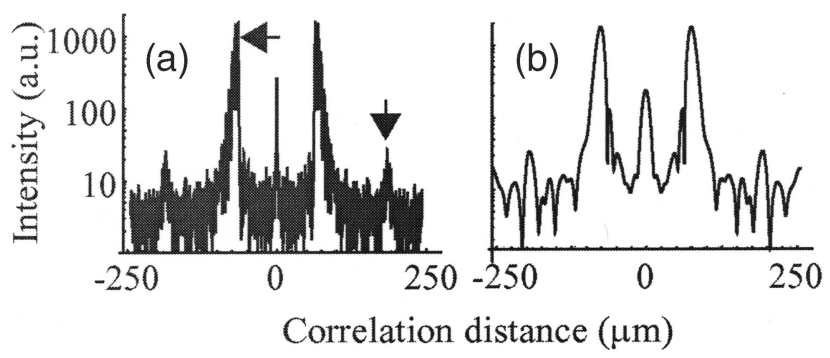

Fig. 3. Axial spatial cross-correlation function for a coverslip sample. (a) High-resolution scan, with arrows indicating the peak corresponding to each glass surface. Note that the correlation function is symmetric about $z=0$, resulting in a superposed mirror image of the scan. (b) Low-resolution scan obtained from scan in (a) by using a Gaussian window. 


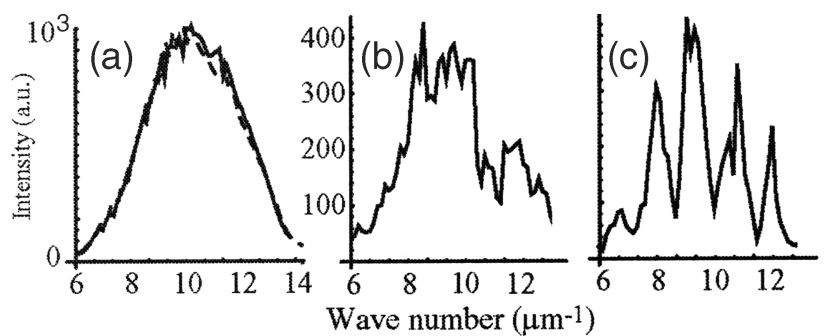

Fig. 4. Spectra obtained for (a) front (solid curve) and (b) back surfaces of the coverglass sample when no microspheres are present. The spectrum of the front surface changes little when microspheres are present [dashed curve in (a)], but in the spectrum of (c) the back surface exhibits periodic modulation.

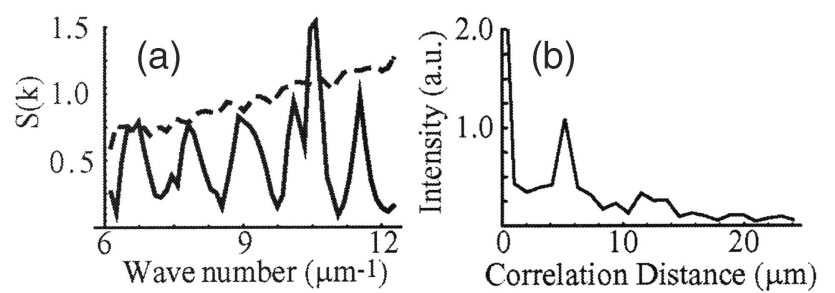

Fig. 5. (a) Ratio of the spectra in Fig. 4, illustrating the scattering efficiency of spheres for front (dashed curve) and back (solid curve) surface reflections. (b) Correlation function obtained from the ratio of back-surface reflections. The peak occurs at the round-trip optical path through individual microspheres, permitting the size of the spheres to be determined with subwavelength accuracy.

the scattered field as $E_{\text {spheres }}(k)=S(k) T(k) E_{s}(k)$ and taking the ratio $E_{\text {spheres }}(k) / E_{\text {back }}(k)=S(k)$. This ratio [Fig. 5(a), solid curve] shows that the microspheres induce a periodic modulation. For comparison, the same ratio for the front-surface reflections [Fig. 5(a), dashed curve] shows only a small linear variation.

Taking the Fourier transform of $S(k)$ yields a clear correlation peak [Fig. 5(b)] at a physical distance of $z=$ $5.24 \mu \mathrm{m} \pm 1.05 \mu \mathrm{m}$. The measurement error arises from uncertainty in the knowledge of the period of the spectral modulation, given by the spectral resolution, $\Delta k_{w}$. The physical distance is related to the optical path length through the sphere by $z=2 n l$, with microsphere index $n=1.59$. We then determine the diameter of the microspheres to be $l=1.65 \mu \mathrm{m}$ $\pm 0.33 \mu \mathrm{m}$. Thus, with fLCI we are able to determine the size of the microspheres with subwavelength accuracy, even exceeding the resolution achievable with this white-light source and conventional LCI imaging.

The anticipated application of fLCI is to determining the sizes of cell organelles, in particular, the cell nuclei, in epithelial tissues. To accomplish this will require several improvements in the setup. In biological media, the relative refractive indices are lower for organelles (1.03-1.06) than for microspheres (1.59), and thus smaller scattering signals are expected. The use of a higher-power light source will permit the smaller signals to be detected. In addition, the larger size of the nucleus than the microspheres in this experiment will result in a higher frequency modulation of the spectrum. It is known that higher frequency oscillations are detected at a lower efficiency with Fourier-transform spectroscopy techniques. ${ }^{13}$ To detect these higher frequency oscillations, a higher-resolution spectrograph will be used.

In conclusion, we have demonstrated that fLCI can recover structural information with subwavelength accuracy from subsurface layers based on measuring elastic-scattering properties. The simplicity of the system makes it an excellent candidate for probing cellular morphology in tissue samples and may one day serve as the basis of a biomedical diagnostic device.

\section{A. Wax's e-mail address is a.wax@duke.edu.}

\section{References}

1. V. Backman, V. Gopal, M. Kalashnikov, K. Badizadegan, R. Gurjar, A. Wax, I. Georgakoudi, M. Mueller, C. W. Boone, R. R. Dasari, and M. S. Feld, IEEE J. Sel. Top. Quantum Electron. 7, 887 (2001).

2. J. R. Mourant, M. Campolat, C. Brocker, O. Esponda-Ramos, T. M. Johnson, A. Matanock, K. Stetter, and J. P. Freyer, J. Biomed. Opt. 5, 131 (2000).

3. A. Wax, C. Yang, V. Backman, K Badizadegan, C. W. Boone, R. R. Darari, and M. S. Feld, Biophys. J. 82, 2256 (2002).

4. I. Georgakoudi, E. E. Sheets, M. G. Müller, V. Backman, C. P. Crum, K. Badizadegan, R. R. Dasari, and M. S. Feld, Am. J. Obstet. Gynecol. 186, 374 (2002).

5. V. Backman, M. B. Wallace, L. T. Perelman, J. T. Arendt, R. Gurjar, M. G. Muller, Q. Zhang, G. Zonios, E. Kline, T. McGillican, S. Shapshay, T. Valdez, K. Badizadegan, J. M. Crawford, M. Fitzmaurice, S. Kabani, H. S. Levin, M. Seiler, R. R. Dasari, I. Itzkan, J. Van Dam, and M. S. Feld, Nature 406, 35 (2000).

6. A. Wax, C. Yang, M. Mueller, R. Nines, C. W. Boone, V. E. Steele, G. D. Stoner, R. R. Dasari, and M. S. Feld, "In situ detection of neoplastic transformation and chemopreventive effects in rat esophagus epithelium using angle-resolved lowcoherence interferometry," Cancer Res (to be published).

7. C. H. Yang, L. T. Perelman, A. Wax, R. R. Dasari, and M. S. Feld, J. Biomed. Opt. 5, 138 (2000).

8. A. Wax, C. H. Yang, R. R. Dasari, and M. S. Feld, Opt. Lett. 26, 322 (2001).

9. A. Wax, C. Yang, V. Backman, M. Kalashnikov, R. R. Dasari, and M. S. Feld, J. Opt. Soc. Am. A 19, 737 (2002).

10. M. Wojtkowski, A. Kowalczyk, R. Leitgeb, and A. F. Fercher, Opt. Lett. 27, 1415 (2002).

11. M. Wojtkowski, R. Leitgeb, A. Kowalczyk, T. Bajraszewski, and A. F. Fercher, J. Biomed. Opt. 7, 457 (2002).

12. R. Leitgeb, M. Wojtkowski, A. Kowalczyk, C. K. Hitzenberger, M. Sticker, and A. F. Fercher, Opt. Lett. 25, 820 (2000).

13. C. Dorrer, N. Belabas, J. P. Likforman, and M. Joffre, J. Opt. Soc. Am. B 17, 1795 (2000).

14. M. D. Kulkarni and J. A. Izatt, in Conference on Lasers and Electro-Optic (CLEO), Vol. 9 of 1996 OSA Technical Digest Series (Optical Society of America, Washington, D.C., 1996), pp. 59-60.

15. U. Morgner, W. Drexler, F. X. Kartner, X. D. Li, C. Pitris, E. P. Ippen, and J. G. Fujimoto, Opt. Lett. 25, 111 (2000). 